\title{
Study of oxide bands in p-type porous silicon layers
}

\author{
Yoselin Rojas-Fernández ${ }^{\mathrm{a}, \mathrm{c}}$, María J. Hernández-López ${ }^{\mathrm{b}}$, Arturo Ramírez-Porras ${ }^{\mathrm{a}, \mathrm{b}, *}$ \\ ${ }^{a}$ CICIMA (Centro de Investigación en Ciencia e Ingeniería de Materiales), Universidad de Costa Rica, San José, Costa Rica \\ ${ }^{\mathrm{b}}$ Escuela de Física, Universidad de Costa Rica, San José, Costa Rica \\ ${ }^{\text {c }}$ Escuela de Ingeniería Química, Universidad de Costa Rica, San José, Costa Rica
}

\section{A R T I C L E I N F O}

\section{Keywords:}

Porous silicon

Impedance spectroscopy

Oxides

\begin{abstract}
A B S T R A C T
The interest in developing fast and reliable chemical and biochemical sensors in an inexpensive way is something that has attracted a lot of efforts in the last decades. One of the potential material candidates is electrochemically treated silicon to form porous silicon (PSi). This treatment generates silicon nanostructures within the porous matrix. The large area to volume ratio is especially interesting for sensing applications. The main drawback of this material is the fast oxidation that impedes the correct device detecting capability.

This work is devoted to the study of the oxidation dynamics in PSi right after preparation. Impedance Spectroscopy (IS) technique was applied on nanocrystalline silicon (NCSi) samples after submitting those surfaces to distinct phases of oxidation. Oxide phases were analyzed by Attenuated Total Reflectance in Fourier Transformed Infrared Spectroscopy (ATR-FTIR) and sessile drop Contact Angle (CA) technique. Nyquist plots were produced and studied by fitting the curves to a model containing a network of passive circuit elements. Results indicate a correlation between oxidation evolution (obtained from ATR and CA data) and the equivalent elements extracted from the impedance model, indicating a change in the surface chemistry of the semiconductor surface. This change is attributed to an increase of $\mathrm{Si}-\mathrm{O}$ bonds in the original surface state.
\end{abstract}

\section{Introduction}

Porous silicon (PSi) was first synthesized in 1956 by Uhlir [1]. This interesting material is obtained usually by electrochemical treatment of a crystalline surface in presence of a strong acid like hydrofluoric acid. Characterizations were performed in a few works in the following decades [2-6]. This material attracted great attention with the discovery of its photoluminescence properties [7]. Since then, many of its properties were tested and research was focused on the development of optic or sensor devices. Very good reviews have been published recently dealing with surface chemistry [8], energy storage [9], photonic sensors [10], and medical applications [11].

Besides all these efforts, in many cases oxidation in PSi negatively impacts the performance of sensing devices as being observed by our group $[12,13]$. A key point to understand the oxidation effect on PSibased sensor devices is to keep track closely on the evolution of the incorporation of oxygen within the porous network. Several studies have shown that for low temperatures (including room temperature), oxygen atoms coming from air moisture breaks Si-Si bonds underneath the silicon surface (passivated with hydrogen atoms) and are incorporated in back bonds to the silicon matrix [14-18]. Therefore, species as $\mathrm{OSi}-\mathrm{H}, \mathrm{O}_{2} \mathrm{Si}-\mathrm{H}$, and $\mathrm{O}_{3} \mathrm{Si}-\mathrm{H}$, as well as $\mathrm{Si-O}-\mathrm{Si}$, are formed while Si-H bonds are relatively stable at those temperatures [19]. FTIR measurements can keep track of this evolution as the material suffers oxidation. On the other hand, Impedance Spectroscopy (IS) is an interesting technique employed to extract information from several systems, mainly in a wet environment [20], although it can be also used in dry systems with some small modifications [21]. Concerning IS applied on PSi, some quite old studies in a wet environment were carried out $[22,23]$, but there is a lack of new information on this topic, especially in a dry environment. The aim of this works is to fill this gap.

\section{Experimental setup}

NCSi samples were obtained using an electrochemical etching method [24]. Boron-doped crystalline silicon substrates of nearly $1 \mathrm{~cm}^{2}$, (100) crystal orientation and $20-50 \Omega \mathrm{cm}$ resistivity, were used as starting material. The etching process was performed using a Teflon cell where the substrates were positioned on the bottom part facing upwards and in contact with an etching solution. The substrate exposed area was a disk of $0.13 \mathrm{~cm}^{2}$. A platinum foil immersed in the solution was used as the cathode, and the back side of the substrate itself was used as the anode. The samples were etched at room temperature in the presence of a solution of ethanoic hydrofluoric acid in the proportion

\footnotetext{
* Corresponding author.

E-mail address: arturo.ramirez@ucr.ac.cr (A. Ramírez-Porras).
} 


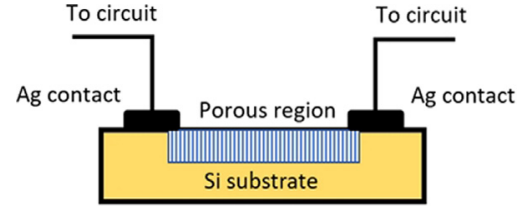

Fig. 1. Schematic cross-section of samples prepared for IS measurements.

[HF:H2O:EtOH] $=1: 5: 2$ per volume $(12.5 \% \mathrm{HF})$. Current density at the constant value of $54 \mathrm{~mA} \mathrm{~cm}^{-2}$ was set using an Agilent 3645 A constant current source. The etching process time was $6 \mathrm{~min}$. After etching, the samples were rinsed with ethanol and deionized water. Silver paste contacts (Leitsilber 200, Ted Pella Inc.) were brushed on opposite sides of the sample in lateral geometry as sketched in Fig. 1. No particular dimensions were set for those contacts, but precautions were taken to have those spots in direct contact with the porous border regions. The samples were inserted into a chamber with a constant flow of dry nitrogen for two hours before performing any measurement to allow silver paste curing at room temperature. Surface characterizations employed were Fourier Transformed Infrared spectroscopy with Attenuated Total Reflectance additament (FTIR-ATR, Frontier model of PerkinElmer employing a diamond crystal in contact with the samples) and Contact Angle measurements (CA using a digital Tucsen CCD camera with Navitar lens and ImageJ software with sessile drop analysis add-in). The used water droplet had a volume of $1 \mu$ l. To perform Impedance Spectroscopy (IS) measurements, a Metrohm Autolab system in potentiostat mode was employed to register Nyquist plots of the samples surfaces with the Nova 2.0 software. Adjusted circuits model parameters were obtained using built-in libraries. Oxidation occurred for the mere contact with ambient air within a term of a few hundreds of minutes.

\section{Results and discussion}

Fig. 2 shows ATR-FTIR spectra for different times of samples air exposure in two regions: $500-1200 \mathrm{~cm}^{-1}$ (right side) and $1800-2300 \mathrm{~cm}^{-1}$ (left side). Important bands are also marked as

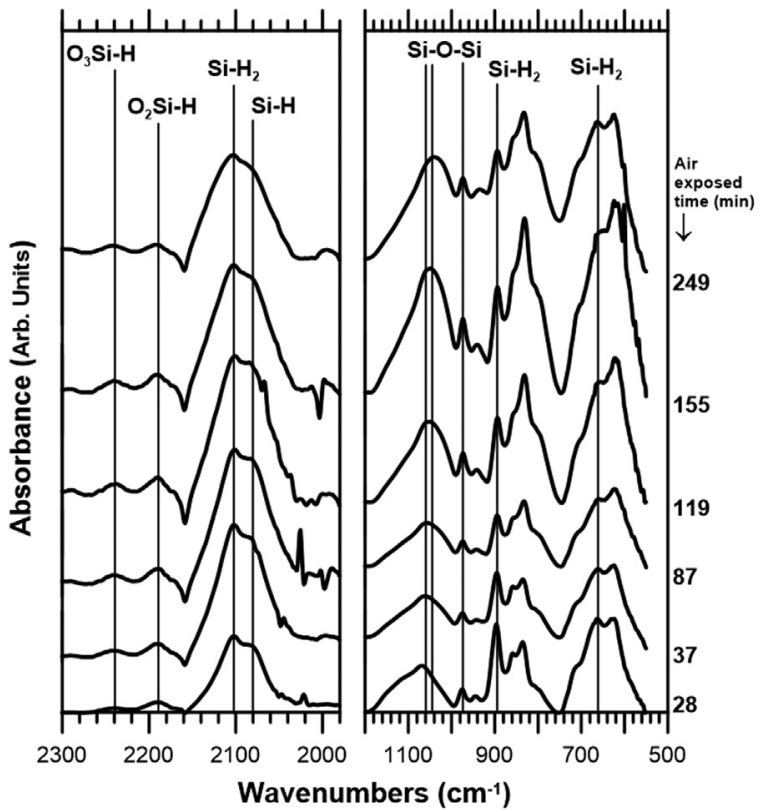

Fig. 2. Evolution of FTIR-ATR for a typical PSi surface in the $500-1200 \mathrm{~cm}^{-1}$ region (right) and $1800-2300 \mathrm{~cm}^{-1}$ region (left). Some important bands are located as vertical lines. The exposed times to ambient air are indicated to the right.

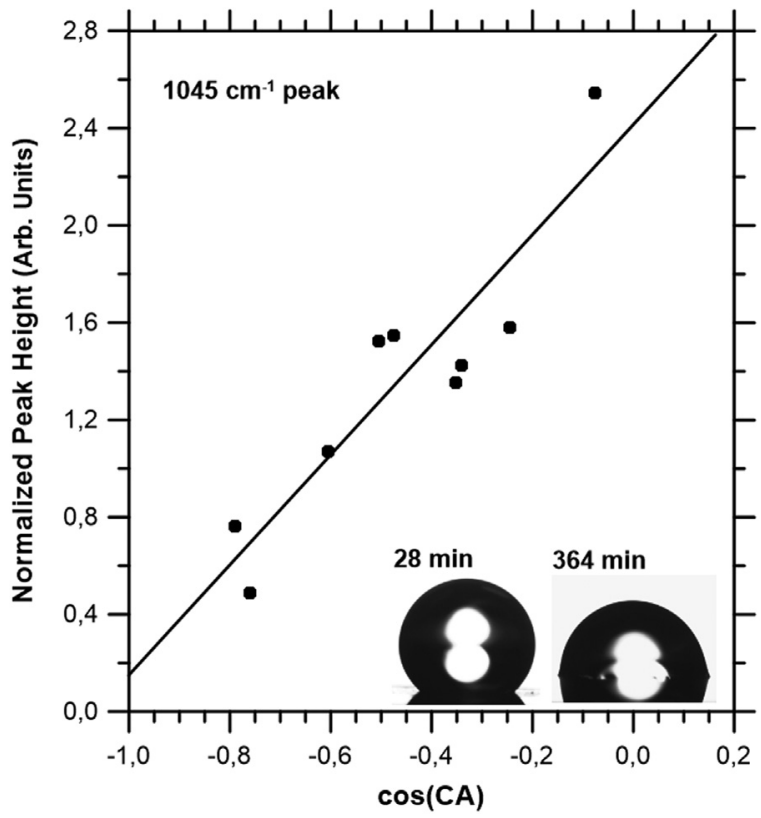

Fig. 3. Normalized SiOSi $1045 \mathrm{~cm}^{-1}$ peak height as a function of the HP. The inset shows two extreme cases of the sessile drop (low air exposure time to the left, and high air exposure time to the right).

vertical lines and correspond to the following. For the right side: $\mathrm{SiH}_{2}$ wagging at $667 \mathrm{~cm}^{-1}$ [18], $\mathrm{SiH}_{2}$ scissors at $902 \mathrm{~cm}^{-1}$ [25], SiOSi asymmetric stretch mode at $980 \mathrm{~cm}^{-1}$ [26], SiOSi stretch TO mode at $1045 \mathrm{~cm}^{-1}$ [25], and SiOSi asymmetric stretch mode at $1060 \mathrm{~cm}^{-1}$ [27]. For the left side: $\mathrm{SiH}$ stretch at $2087 \mathrm{~cm}^{-1}, \mathrm{SiH}_{2}$ stretch at $2108 \mathrm{~cm}^{-1}, \mathrm{O}_{2} \mathrm{SiH}$ stretch at $2200 \mathrm{~cm}^{-1}$, and $\mathrm{O}_{3} \mathrm{SiH}$ stretch at $2256 \mathrm{~cm}^{-1}$ [18]. All these lines have been identified in the literature as important to study oxidation in silicon surfaces.

Spectra peaks were normalized to the $902 \mathrm{~cm}^{-1}$ (right plot) and $2108 \mathrm{~cm}^{-1}$ (left plot) $\mathrm{SiH}_{2}$ modes to enhance the change in the silicon oxide bands. All normalizations, including baseline definitions, were performed using the built-in libraries of the PerkinElmer Spectrum software. As time evolves, it appears that the $\mathrm{Si}-\mathrm{O} / \mathrm{Si}-\mathrm{H}$ ratio increases. This behavior has been observed previously for oxidation processes in anodized silicon surfaces [14]. To quantify this fact, a deconvolution into all those vibrational bands was performed using PeakFit 4.12 software (Systat Software Inc.). Fig. 3 shows the relation between the peak height of SiOSi bonds at $1045 \mathrm{~cm}^{-1}$ against the hydrophilic parameter (HP), defined as the cosine of the contact angle. If HP is negative, the surface is hydrophobic, whereas if it is positive, it tends to be hydrophilic. A similar trend (not shown here) has been obtained for the $\mathrm{O}_{3} \mathrm{Si}-\mathrm{H} 2245 \mathrm{~cm}^{-1}$ peak as a function of HP. In Fig. 3, a direct relationship is clearly visible, indicating that HP can be used as a quick measure of hydrophilicity and, consequently, of oxidation progress. This detail simplifies the determination of oxide evolution for the next analysis step, where the sample must be immobilized and therefore it cannot be taken to the FTIR spectrometer, but the determination of CA is easy to be performed in place.

We must point out that the two-hour drying process in a nitrogen atmosphere did not change HP appreciably $(-0.620$ from fresh sample to -0.661 after $\mathrm{N}_{2}$ drying). Consequently, we expected that no appreciable oxidation showed up during the drying period.

In Fig. 4, a series of Nyquist plots are sketched for times that run right after extraction of the sample from the $\mathrm{N}_{2}$ ambient (marked as "0 min") to $165 \mathrm{~min}$ in contact with air. In those plots, frequency increases counterclockwise. The half circular shape of plots is typical of moving and storing charges in the system [20]. The interception of the curves with the horizontal axis shifts towards higher values, indicating an increase in static resistance within the device. This outcome is 


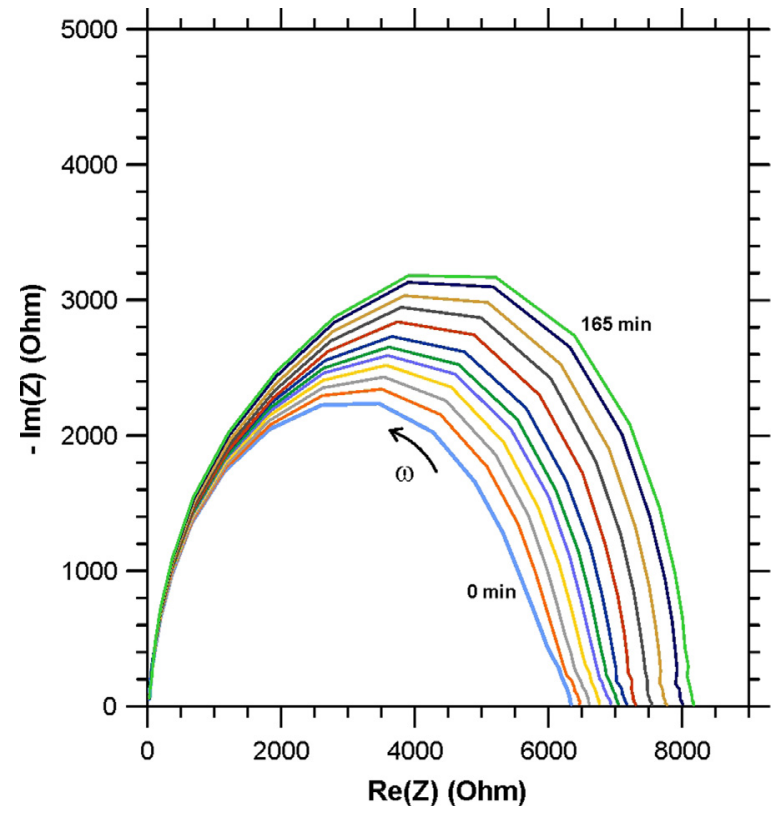

Fig. 4. Nyquist plots for a PSi sample. The shown labels mark two extreme cases of measurements: first measurement right after taking out the sample from $\mathrm{N}_{2}$ $(0 \mathrm{~min})$ and the last measurement performed $(165 \mathrm{~min})$. The arrow shows the increasing frequency way.

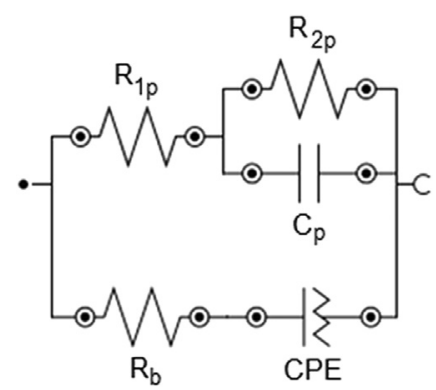

Fig. 5. Circuit model for the IS technique. All resistance, capacitance and CPE parameters can be fit for the different curves shown in Fig. 4.

similar to the previously observed in a wet environment for aged samples $[16,28]$.

The system behavior can be modeled by a network of resistors, capacitors and constant phase elements (CPE), which are essentially capacitors with losses [21,29]. Fig. 5 sketches the equivalent circuit model that fits the data curves shown in Fig. 4. $R_{1 p}$ and $R_{2 p}$ represent the porous surface resistances, $R_{b}$ the substrate resistance, $C_{p}$ a parallel capacitor contribution in the porous network, and the CPE models possible changes in the flow of charge between substrate and contacts in the Schottky barrier, which is not necessarily linear [30]. From CPE fit parameters, it is still possible to calculate an equivalent capacitance $\mathrm{C}_{\mathrm{eq}}$ according to the procedure mentioned in Ref. [29].

Fig. 6 plots the resulting capacitances and resistances of the circuit model shown in Fig. 5 for increasing oxidation measured using the adjusted HP extracted by the linear fit shown in Fig. 3. The resulting values for the substrate and contacts contributions are fairly constant: $\mathrm{R}_{\mathrm{b}} \approx 24 \Omega$ and $\mathrm{C}_{\mathrm{eq}} \approx 4.2 \mathrm{nF}$. This was expected because those regions are not being targeted for oxidation. On the contrary, the parameters modeling what happens at the porous surface change with oxidation: $\mathrm{C}_{\mathrm{p}}$ decreases from $79 \mathrm{nF}$ to $17 \mathrm{nF}$ (78\% drop), $\mathrm{R}_{2 \mathrm{p}}$ increases from $1.2 \mathrm{k} \Omega$ to $2.6 \mathrm{k} \Omega$ ( $117 \%$ higher), and $\mathrm{R}_{1 \mathrm{p}}$ slightly from $4.9 \mathrm{k} \Omega$ to $5.5 \mathrm{k} \Omega(12 \%$ higher). A rise in resistance is expected since the incorporation of oxygen atoms within the semiconductor network (change from $\mathrm{Si}-\mathrm{Si}$ bonds to $\mathrm{Si}-\mathrm{O}$ bonds as explained previously) breaks charge transport

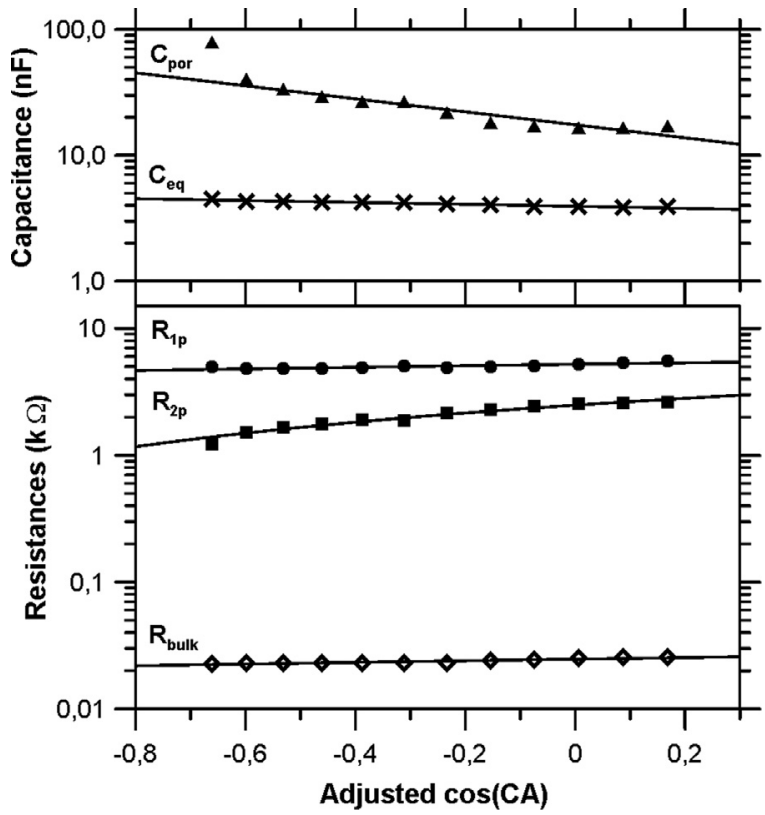

Fig. 6. Extracted parameters from the fitting procedure as a function of the HP (equivalent capacitances in the upper plot; substrate and porous surface resistances in the lower plot).

channels, replacing them by more isolating regions (in an extreme case, a fully surface oxidation would produce vitrification of the pores). These observations agree with an increase in the surface oxide coverage in silicon clusters reported previously but using a different methodology [12]. In the case of the porous capacitance, we assume that it is related to the inverse of the depletion layer width near the surface of the semiconductor [30], so, therefore, it must be influenced by the chemical ambient in that region. We propose that the depletion layer width increases by virtue of the oxide layer that grows with time, so it is plausible that the equivalent geometric porous capacitance decreases as a result. Nevertheless, this proposal should be tested more exhaustively in a near future.

\section{Conclusions}

A direct relationship between oxide bonds obtained by FTIR-ATR and CA measurements advised us to use CA as a rapid methodology to estimate the degree of oxidation in porous silicon surfaces. This feature can improve the analysis of aging on this kind of surfaces without the need to extract the material from a device for characterization needs. On the other hand, the equivalent circuit model arising from IS measurements results in an increase of resistance in the porous region as well as a decrease in its geometric capacitance. The reasons for these behaviors come from the possible growth in oxide thickness within the porous matrix.

\section{Acknowledgments}

The contact author acknowledges the support of the Vicerrectoría de Investigación of the Universidad de Costa Rica, Costa Rica, under the research project no. 816-B6-146.

\section{References}

[1] A. Uhlir, Bell Syst. Tech. J. 35 (1956) 333-347.

[2] D.R. Turner, J. Electrochem. Soc. 105 (1958) 402-408.

[3] G. Bomchil, R. Herino, K. Barla, J.C. Pfister, J. Electrochem. Soc. 130 (1983) 1611-1614.

[4] K. Barla, R. Herino, G. Bomchil, J.C. Pfister, A. Freund, J. Cryst. Growth 68 (1984) 727-732. 
[5] M.I.J. Beale, N.G. Chew, M.J. Uren, A.G. Cullis, J.D. Benjamin, Appl. Phys. Lett. 46 (1985) 86-88.

[6] V. Labunov, V. Bondarenko, L. Glinenko, A. Dorofeev, L. Jabuhna, Thin Solid Films 137 (1986) 123-134.

[7] L.T. Canham, Appl. Phys. Lett. 57 (1990) 1046-1048.

[8] S.H. Lee, J.S. Kang, D. Kim, Materials 11 (2018) 2557.

[9] R.Z.A. Manj, X. Chen, W.U. Rehman, G. Zhu, W. Luo, J. Yang, Front. Chem. 6 (2018) 539.

[10] S. Arshavsky-Graham, N. Massad-Ivanir, E. Segal, S. Weiss, Anal. Chem. 91 (2019) $441-467$.

[11] T. Tieu, M. Alba, R. Elnathan, A. Cifuentes-Rius, N.H. Voelcker, Adv. Ther. 2 (2019) 1800095.

[12] C. Vargas, A. Corrales, T. Ramírez-Cortés, A. Ramírez-Porras, Phys. Status Solidi C 12 (2015) 1383-1386.

[13] C. Vargas, T. Ramírez-Cortés, K. Cordero-Solano, A. Ramírez-Porras, MRS Adv. (2017) 276.

[14] Y. Kato, T. Ito, A. Hiraki, Jpn. J. Appl. Phys. 27 (1988) L1406-L1409.

[15] P. Gupta, V.L. Colvin, S.M. George, Phys. Rev. B 37 (1988) 8234-8242.

[16] V. Parkhutik, Solid-State Electron. 43 (1999) 1121-1141.

[17] D.D.M. Wayner, R.A. Wolkow, J. Chem. Soc. Perkin Trans. 2 (2002) 23-34.

[18] Y.H. Ogata, Characterization of porous silicon by infrared spectroscopy, in:
L. Canham (Ed.), Handbook of Porous Silicon, Springer, Cham, 2014.

[19] S. Sinha, N.W. Piekiel, G.L. Smith, C.J. Morris, Combust. Flame 181 (2017) 164-171.

[20] A.J. Bard, L.R. Faulkner, Electrochemical Methods, Fundamentals and Applications, John Wiley \& Sons, Inc, New York, 2001.

[21] R. Schmidt, Impedance spectroscopy of electroceramics, in: P.B. Lin (Ed.), Ceramic Materials Research Trends, Novascience Publishers, Hauppauge, 2007, p. 321.

[22] V.P. Parkhutik, E.S. Matveeva, R. Díaz Calleja, Electrochim. Acta 41 (1996) 1313-1321.

[23] V.P. Parkhutik, J. Porous Mater. 7 (2000) 97-101.

[24] A. Ramírez-Porras, O. García, C. Vargas, A. Corrales, J.D. Solís, Appl. Surf. Sci. 347 (2015) 471-474

[25] F.A. Rustamov, N.H. Darvishov, V.E. Bagiev, M.Z. Mamedov, G.M. Eyvasova, E.Y. Bobrova, H.O. Qarafova, J. Lumin. 195 (2018) 49-53.

[26] S.M. Iftiquar, High. Temp. Mater. Proc. 6 (2002) 35-54.

[27] C.A. Caras, J.M. Reynard, R.E. Deuro, F.V. Bright, Appl. Spectrosc. 66 (2012) 951-957.

[28] V. Parkhutik, E. Matveeva, Phys. Status Solidi A 182 (2000) 37-44.

[29] C.H. Hsu, F. Mansfeld, Technical note: concerning the conversion of the constant phase element parameter $Y_{0}$ into a capacitance, Corrosion 57 (2001) 747-748.

[30] S.M. Sze, Physics of Semiconductor Devices, John Wiley \& Sons, New York, 1981. 